\title{
Unleashing regenerative potential
}

The potential to replace damaged tissues, organs and appendages is obviously of great biomedical interest, but, unfortunately, not all organisms have extensive regenerative abilities. New work shows that the Wnt- $\beta$-catenin pathway is crucial to the regeneration of appendages in several vertebrates. Remarkably, the regenerationpromoting properties of $\beta$-catenin function are not restricted to organisms that are naturally able to regrow appendages. This molecule can also promote limb regeneration in the chick embryo, in which limb regeneration was not previously thought to occur.

The authors first showed in axolotls, Xenopus laevis and zebrafish that interfering with the Wnt pathway through infection with adenoviruses expressing antagonists such as Axin1 or Dkk1 caused defects in limb or fin regeneration. Consistent with this, expression of an activated form of $\beta$-catenin was able to promote limb regeneration in $X$. laevis, and to rescue a zebrafish mutation with defective regenerative properties.

Disruption of Wnt signalling alters the formation of the apical ectodermal cap (AEC) - the multilayered epithelium that covers the wound after amputation - and this defect correlates with changes in the spatio-temporal distribution of p63. Interestingly, p63 is required in higher vertebrates for the formation of the apical ectodermal ridge (AER), a pseudostratified epithelium that, similar to the regenerating AEC, drives the proliferation of mesenchymal cells to shape the developing limb.

Given the similarities of these processes, Kawakami and colleagues used a non-regenerating animal, the chick, to investigate the role of the Wnt pathway following AER removal. They found that activated $\beta$-catenin had the ability to rescue the expression of $f g f 8$, a member of the fibroblast growth factor (FGF) family that is involved in limb outgrowth, and can induce the regeneration of the AER, which is essential for normal limb development.

So, regeneration in adults and tissue generation during embryonic development seem to share similar mechanisms. A further understanding of this overlap could allow regeneration to be stimulated in other tissues and structures that are not normally able to regenerate, which would have invaluable medical applications.

Francesca Pentimalli

\section{ORIGINAL RESEARCH PAPER}

Kawakami, Y.et al. Wnt/ $\beta$-catenin signaling

regulates vertebrate limb regeneration. Genes Dev.

17 November 2006 (doi:10.1101/gad.1475106) FURTHER READING Alvarado, A. S. \& Tsonis, P. A. Bridging the regeneration gap: genetic insights from diverse animal models. Nature Rev. Genet. 7 , 873-884 (2006)

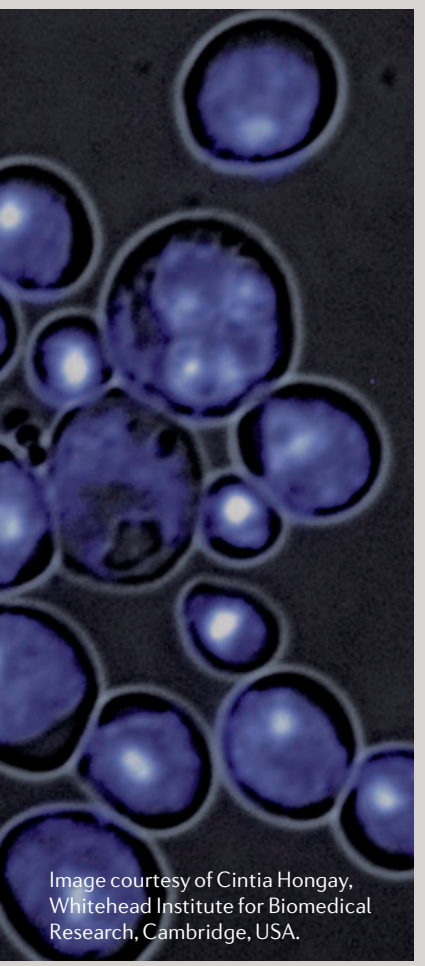

RNA WORLD

\section{Haploids and diploids make no sense of each other}

Yeast cells do not regulate gene expression by RNAi, yet they do transcribe various non-coding RNAs. A new study shows that one of the key decisions in the yeast life cycle - entry into meiosis - is determined by a non-coding antisense RNA that interferes with sense transcription.

Mating in Saccharomyces cerevisiae occurs between haploid cells of opposite mating type - $\mathbf{a}$ and $\alpha$. In the resultant diploid cell, haploid functions are repressed by the $a 1-\alpha 2$ protein heterodimer. Under certain conditions, the diploid will initiate meiosis, which requires transcription of several genes including IME4 (initiator of meiosis 4). Transcription of this gene is repressed in haploids, and now Gerald Fink and colleagues show how.
The authors found that haploids and diploids expressed transcripts from the IME4 locus that differ in both their length and the strand that is transcribed. In the haploid cells, an antisense transcript is made that encompasses the entire region that is transcribed from the sense strand in diploids. They then showed that a1- $\alpha 2$ binds to a site that lies upstream of the initiation site of the antisense transcript and represses its transcription. Diploids that are homozygous for a mutation in this site transcribe the antisense strand rather than the sense strand, and have haploid-like phenotypes. Conversely, haploids that have been engineered to express both components of a $1-\alpha 2$ transcribe the sense strand.
In theory, this antisense interference effect could occur through various mechanisms, including something similar to RNAi. However, the authors show that the antisense repression occurs only in cis and is therefore likely to be caused by transcriptional interference. Neither heterozygotes for the mutated a1- $\alpha 2$-binding site nor diploids that were engineered to express sense and antisense transcripts from different loci showed repression of sense transcription. This implies that in the wild-type situation transcription of the sense transcript is only impeded by transcription in the opposite direction at the same locus on the same chromosome. As the antisense promoter is stronger, this is the dominant transcript 


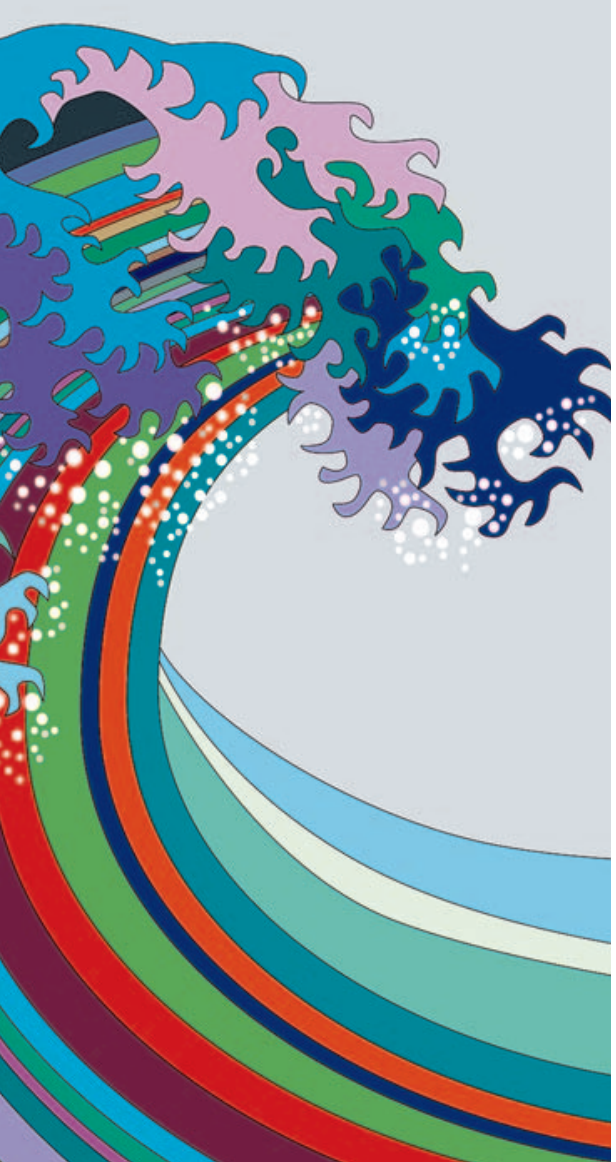

in haploids; in diploids, sense transcription can occur because antisense transcription is repressed by a $1-\alpha 2$. Consistent with this, when the sense transcript is artificially overexpressed in haploids by substituting a stronger promoter, antisense transcription is abolished.

Although these results show how yeast make use of non-coding RNA in gene regulation, the antisense mechanism is different from the RNA $i$ that is used in higher eukaryotes. It is likely that a similar mechanism will apply to the other antisense transcripts that have been detected in yeast. Furthermore, they might also apply to mammals, in which antisense transcripts have also recently been detected.

Patrick Goymer

\section{ORIGINAL RESEARCH PAPER}

Hongay, C. F. et al. Antisense transcription controls cell fate in Saccharomyces cerevisiae. Cell 127, 735-745 (2006)

\section{$\Rightarrow$ PATHOGEN GENOMICS}

\section{Parasite diversity promises malaria secrets}

Three new studies provide the most detailed description yet of the genetic diversity in the genome of the parasite that causes malaria Plasmodium falciparum - and shed light on its recent evolutionary history. These advances pave the way for new approaches to understanding key features of the biology of this parasite and new avenues for combating infection.

Volkman, Sabeti and colleagues generated a map of genetic diversity across the P. falciparum genome. They sequenced 16 strains from a range of geographical locations, identifying almost 47,000 variants (SNPs) and revealing a high degree of diversity among strains. The authors also studied linkage disequilibrium (LD) patterns between SNPs in 20 genomic regions.

LD between SNPs can be put to use in mapping studies that look for association between variants and phenotypes of interest to identify the genes that are involved. The greater the extent of LD between SNPs, the fewer need to be genotyped to cover the whole genome in such studies. The authors found that parasites from Asia show more extensive LD between SNPs than those from Africa - so more SNPs are likely to be needed for comprehensive association studies in African parasites.

In the same study, the authors looked for genomic regions with lower than average diversity in specific strains, indicating that genes within these regions have been subject to recent strong directional selection. This approach suggested several candidate regions that might have been subject to selection due to drug exposure. Promisingly, one contains the pfcrt locus, which is known to be involved in chloroquine resistance.

In a second study, Mu, Awadalla and colleagues also surveyed variation in the P. falciparum genome. Using isolates from around the world, they generated a combined genomewide map of polymorphic microsatellites and SNPs, with an average of one marker every $\sim 3.6 \mathrm{~kb}$ - another valuable resource for mapping studies.

This group also searched for signs of balancing selection - which is a feature of genomic regions that contain antigen-encoding genes - indicated by the presence of polymorphisms in consecutive genes. This approach identified a large number of candidate regions, leading the authors to identify several previously uncharacterized genes that encode antigens, which they confirmed using human immune sera.

In a third study, Jeffares and colleagues characterized both genetic variation among $P$. falciparum isolates and between this species and its closest relative that does not infect humans, Plasmodium reichenowi. Again, a high level of diversity in P. falciparum was revealed, as well as a tenfold greater level of genetic difference with $P$. reichenowi. The authors identified regions with signatures of positive selection by comparing ratios of synonymous and non-synonymous substitution rates, and genes that are likely to have undergone directional or diversifying selection by assessing levels of divergence or polymorphism. As well as known antigen-encoding genes, the resulting list contains a number of genes of as yet unknown function, providing intriguing new candidate antigens.

The marker sets that are provided by these studies, and the information they reveal about LD patterns and allele frequencies, provide a strong basis for association mapping in P. falciparum. This promises to circumvent the current limitations of mapping in this species due to the difficulty of carrying out traditional genetic studies. As the examples that are described above indicate, searches for signatures of selection should also provide fertile ground for identifying urgently needed new candidates for therapeutic strategies.

Louisa Flintoft

\section{ORIGINAL RESEARCH PAPERS}

Jeffares, D. C. et al. Genome variation and evolution of the malaria parasite Plasmodium falciparum. Nature Genet. 10 December 2006 (doi:10.1038/ng1931) | Volkman, S. K. et al. A genome-wide map of diversity in Plasmodium falciparum. Nature Genet. 10 December 2006 (doi:10.1038/ng1930) | $\mathrm{Mu}$, J. et al. Genome-wide variation and identification of vaccine targets in the Plasmodium falciparum genome. Nature Genet. 10 December 2006 (doi:10.1038/ng1924)

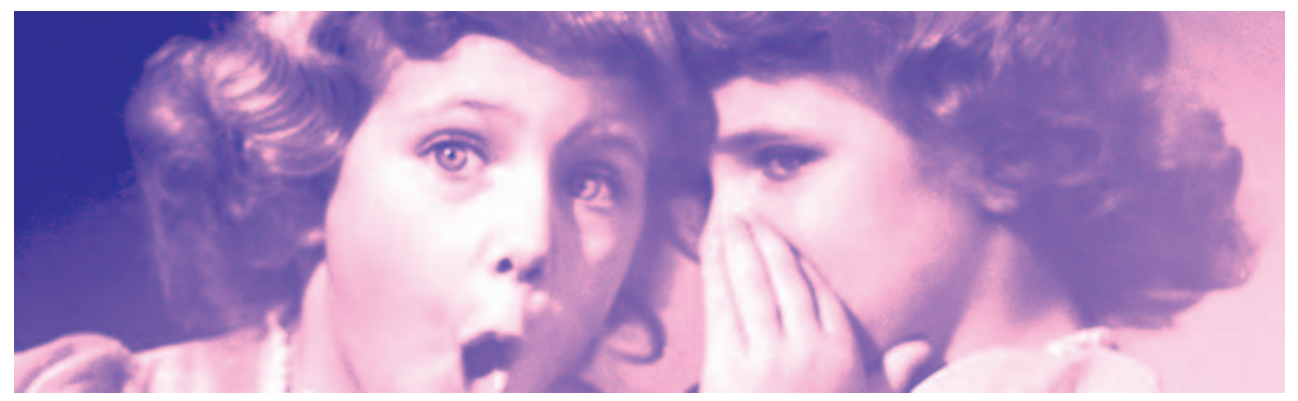

